\title{
AVALIAÇÃO DA FORÇA MUSCULAR DO ASSOALHO PÉLVICO EM MULHERES SEDENTÁRIAS E QUE PRATICAM ATIVIDADE FÍSICA
}

\author{
Tereza Cristina dos Reis FERREIRA ${ }^{1}$ \\ Amanda Alves GODINHO ${ }^{2}$ \\ Amanda Rabelo de MELO ${ }^{3}$ \\ Rayanne Tortola REZENDE ${ }^{4}$
}

\author{
${ }^{1}$ UEPA - Departamento do Movimento Humano-Curso de Fisioterapia. CESUPA - Áreas das Ciências \\ Ambientais,Biológicas e da Saúde-Curso de Fisioterapia. tereza_reis@ yahoo.com.br \\ ${ }^{2}$ Centro Universitário do Pará. aamandagodinho@ gmail.com \\ ${ }^{3}$ Centro Universitário do Pará. amandar_m@ hotmail.com \\ ${ }^{4}$ Centro Universitário do Pará. rayanne tortola@ hotmail.com
}

Recebido em: 12/06/2015 - Aprovado em: 15/09/2015 - Disponibilizado em: 30/10/2015

\begin{abstract}
RESUMO
Atualmente pessoas que praticam exercícios físicos em academias vêm crescendo. Entretanto, um grande número de mulheres sedentárias, e que praticam atividade física, demonstram desconhecimento acerca da importância de realizar o fortalecimento do assoalho pélvico durante toda a vida, pois esta musculatura sustenta órgãos importantes. Objetivo da pesquisa é comparar o grau de força muscular do assoalho pélvico entre os grupos de mulheres sedentárias e de mulheres praticantes de atividade física nas acadêmicas de Belém do Pará. A amostra em estudo é do tipo intencional, constituída por 10 mulheres que praticam atividade física e sedentárias, com faixa etárias 20 a 35 anos, submetidas à anamnese e avaliação da força muscular do assoalho pélvico, foram adotados dois meios de avaliação, o AFA, e pelo aparelho Myofeedback com o recurso do biofeedback. Obtiveram-se os resultados do teste de Mann- Whitney com o p-valor $=0.0275$, observando que a força muscular das mulheres sedentárias é inferior às praticantes. No resultado do $\mathrm{p}$-valor $=0.0105$ identificou que a relação entre a idade e a força foi significante, mostrando que conforme a idade for aumentando ocorre uma alteração da força muscular. De acordo com p-valor $=0.0105$ e p-valor $<0.005$, pode-se observar que o peso também pode interferir na força do assoalho pélvico. Verificou-se, que há diferença de força, entre mulheres sedentárias e praticantes, pois as mulheres do grupo de praticantes, obtiveram resultados superiores de grau de força durante a avaliação, sendo então observado diferentes tipos de força, como AFA, força máxima e resistência.
\end{abstract}

Palavras-Chave: Fisioterapia. Assoalho Pélvico. Atividade Física. Sedentárias. Biofeedback.

\section{MUSCLE STRENGTH ASSESSMENT THE PELVIC FLOOR IN SEDENTARY WOMEN AND PRACTICING PHYSICAL ACTIVITY}

\begin{abstract}
Currently people who practice physical exercises in gyms are growing. However, a large number of sedentary women, who practice physical activity, demonstrate ignorance about the importance of holding strengthening the pelvic floor throughout life, as this muscle sustains major organs. The search goal is to compare the degree of muscle strength of the pelvic floor between the groups of sedentary women and women engaged in physical activity in Belém do Pará academic. The sample in question is intentional, consisting of 10 women who practice physical activity and sedentary with age range 20-35 years submitted to an interview and evaluation of muscular strength of the pelvic floor, were adopted two means of evaluation the AFA, and the Myofeedback device with biofeedback feature. Were obtained the results of the Mann-Whitney test with p-value $=0.0275$, noting that the muscle strength of sedentary women is lower than practitioners. The result $\mathrm{p}$-value $=0.0105$ which identified the
\end{abstract}


relationship between age and the strength was significant, showing that with age there is a change for increasing muscular strength. According $\mathrm{p}=0.0105$ and $\mathrm{p}<0.005$, it can be seen that the weight can also interfere with the strength of the pelvic floor. It was found that there is difference in strength between sedentary women and practitioners, since women's group practitioners, obtained better results than the degree of strength during the assessment, and then observed different types of force, as AFA, maximum strength and endurance.

Keywords: Physiotherapy . Pelvic floor . Physical Activity. Sedentary . Biofeedback.

\section{INTRODUÇÃO}

Atualmente o número de pessoas que exercem a prática de exercícios físicos em academias vem crescendo consideravelmente, pois a atividade física é de fundamental importância para o ser humano, diminuindo a probabilidade de desenvolver doenças como obesidade, osteoporose, doenças coronarianas, entre outras (SILVA, MORAES 2006).

Segundo Silva e Leme (2012) algumas pessoas optam por modalidades esportivas de alto impacto como voleibol, basquete, saltos, corridas, enquanto outras por atividades aeróbias de baixo impacto. Alguns estudos têm demonstrado que mulheres atletas apresentam disfunção do assoalho pélvico devido ao alto impacto da atividade física, que poderia promover uma sobrecarga dinâmica nas estruturas internas da região abdomino-pelvica, desencadeando sintomas uroginecológicos. Porém, pouco se sabe qual a verdadeira influência das atividades esportivas como fator de risco nas disfunções uroginecológicas.
Existe, entretanto, um grande número de mulheres que não realizam nenhum tipo de atividade física, denominadas sedentárias, e tanto estas, quanto as que praticam atividade, demonstram desconhecimento acerca da importância de exercícios para fortalecer a musculatura do assoalho pélvico (SILVA, MORAES, 2006).

Silva e Moraes (2006) relata que a mulher deveria fazer exercícios para o fortalecimento do assoalho pélvico durante toda a vida, pois esta musculatura sustenta órgãos muito importantes e não pode ser esquecida.

$$
\text { O Assoalho Pélvico }
$$
representa o conjunto das partes moles que tem sua composição músculo esquelética, onde sua função é sustentar os órgãos pélvicos, fechar a pelve e suportar as vísceras em posição vertical, manter a continência urinária e fecal, sendo também imprescindível para a função sexual, na gravidez e no parto. Este conjunto consiste de músculos, ligamentos e fáscias, dispostos de modo a proporcionar uma ação esfincteriana para a uretra, reto, e na 
mulher, vagina, além de permitir a passagem de um feto a termo. É dividido em três compartimentos: anterior (bexiga e uretra), médio (vagina) e posterior (reto) (ZUCCHI, 2003; MORENO, 2004).

Segundo a Sociedade Internacional de Continência, Incontinência Urinária (IU) é definida como perda involuntária de urina. Os tipos mais comuns são: a incontinência urinária de esforço (IUE), que é a perda de urina associada com atividades físicas que aumentam a pressão intrabdominal, à incontinência urinária de urgência (IUU), perda involuntária de urina associada a um forte desejo de urinar, e a incontinência urinária mista (IUM), quando ambos os tipos anteriores estão presentes (VIRTUOSO, 2011).

Segundo Almeida et al (2011), autores relatam a prevalência de IU em jovens atletas, muitas vezes nulíparas, e concluíram que o esporte de treinamento intensivo é um fator de risco independente para IU. As condições que contribuem para as doenças de assoalho pélvico em atletas jovens e nulíparas ainda não estão completamente esclarecidas. A hipótese é que atividades esportivas levam ao frequente aumento da pressão intraabdominal, podendo levar à fadiga ou ao dano das estruturas musculares e conectivas do AP.
A Incontência Urinária vem crescendo consideravelmente. Aproximadamente 200 milhões de pessoas no mundo apresentam algum tipo de IU, o mais frequente tipo e a incontinência urinária de esforço (IUE), definida como perda involuntária de urina mediante um esforço físico, espirro, tosse ou atividade física (PINHEIRO, SOUZA, 2011).

Segundo Fitz (2012) a prevalência dos sintomas da Incontinência Urinária de Esforço (IUE) de qualquer intensidade é de aproximadamente $80 \%$ em mulheres entre 25 e 60 anos de idade. Dentre as modalidades esportivas, a taxa de prevalência da IUE varia de $10 \%$ na natação (modalidade de baixo impacto), chegando ate $40 \%$ para o atletismo e atividades aeróbicas (alto impacto, como o voleibol), levando $20 \%$ dessas esportistas a abandonarem suas atividades e $40 \%$ a mudarem de modalidade (BORIN, 2006).

Em atletas a força da musculatura abdominal tende a ser alta, isso pode ser tanto devido a modalidade esportiva que exija essa musculatura, quanto devido o treinamento físico geral que estas são submetidas. Se houver fraqueza da musculatura do assoalho pélvico em relação às situações citadas, podem ocorrer episódios de perda urinária quando submetidas ao esforço físico, mesmo na 
ausência dos outros fatores de riscos (REIS et al, 2011).

Nesse contexto, a fisioterapia tem como objetivos a prevenção, cura, reabilitação e reeducação de uma gama variada de patologias, é de fundamental importância, pois irá melhorar a percepção da musculatura do assoalho pélvico, melhorar a força de contração das fibras musculares e estimular bons hábitos de vida, como cita Berquó (2009).

A avaliação da contração voluntária dos Músculos do Assoalho Pélvico (MAP), envolve a habilidade de elevar o AP, tanto quanto a manutenção da força, endurance e coordenação muscular, através da manutenção e contração da musculatura perineal, ou seja, da força apresentada pela mesma, que pode-se avaliar sua funcionalidade na sustentação dos órgãos pélvicos, na geração e manutenção da pressão positiva uretral feminina. (MOREIRA; ARRUDA, 2010).

A atividade física em academias inclui exercícios de contração abdominal máxima de forma repetitiva, mimetizando assim, o desempenho de atletas e os efeitos causados sobre os músculos do assoalho pélvico na realização de esportes.

Dessa forma, a fim de verificar se há diferença dessa força muscular entre os grupos de mulheres sedentárias e aquelas praticantes de atividade física, esta pesquisa tem como objetivo geral comparar o grau de força muscular do assoalho pélvico entre os grupos de mulheres sedentárias e de mulheres praticantes de atividade física nas academias de Belém do Pará.

\section{METODOLOGIA}

A presente pesquisa respeitou os preceitos da Declaração de Helsinque e do Código de Nuremberg, preconizando as Normas de Pesquisa Envolvendo Seres Humanos (Res. CNS 466/12) do Conselho Nacional de Saúde. Teve início após o aceite da orientadora, posterior à submissão e aprovação do projeto pelo Comitê de Ética em pesquisa do Centro Universitário do Estado do Pará, sob o parecer de numero CAAE 0079.0.323.00013, posterior à autorização do local de estudo, a Clínica Escola de Fisioterapia do CESUPA, Belém - PA.

Trata-se de um trabalho do tipo quantitativo, estatístico descritivo e exploratório. Onde se aplicou o uso do Termo de Consentimento Livre e Esclarecido (TCLE) que foi entregue e explicado para cada participante no primeiro momento do estudo, antes de qualquer intervenção fisioterapêutica 
A amostra foi do tipo intencional, constituída por aproximadamente 10 mulheres que praticam atividade física e que não praticam, com faixa etária entre 20 a 35 anos.

Foram aplicados critérios para que as mulheres pudessem ou não ser inseridas na pesquisa. Critérios de inclusão: praticantes de atividade física e não praticantes, do sexo feminino, com idade de 20 á 35 anos, as que realizam atividade física que atuavam a pelo menos 6 meses, com frequência de três treinos semanais, duração de no mínimo 1 hora cada treino, que não faziam o uso de medicamento, exceto anticoncepcional, com atividade sexual iniciada.

Foram excluídas praticantes de atividade física e não praticantes, com diagnóstico ou sequela de doenças ginecológicas, gestantes, usuárias de medicamentos (exceto anticoncepcional) e as que utilizavam marca passo, ou seja, cardíacas.

A pesquisa iniciou-se com uma triagem, realizada com todas as mulheres da pesquisa, que consistia em dados e antecedentes pessoais, antecedentes ginecológicos e obstétricos, hábitos e antecedentes familiares, e questão sobre incontinência urinária e atividade física, elaborado pelas autoras.
Posteriormente as participantes foram encaminhadas para realização da coleta dos dados do exame físico, que consta com, sinais vitais, como Pressão Arterial (PA), Frequência Respiratória (FR), Frequência Cardíaca (FC), Saturação Periférica de $\mathrm{O} 2$ (SpO2), Peso, Altura, Índice de Massa Corpórea (IMC).

Para a avaliação da força muscular do assoalho pélvico foram adotados dois meios de avaliação, o AFA (Avaliação da força do assoalho pélvico), e pelo aparelho Myofeedback da marca Quark modelo Perina com o recurso do biofeedback manométrico-perineal, onde a pressão foi mensurada em $\mathrm{mmHg}$.

Ao iniciar os testes, foi solicitado, que a paciente se posicionasse deitada em decúbito dorsal, com flexão de quadril de aproximadamente $60^{\circ}$ graus e flexão de joelho de aproximadamente $45^{\circ}$ graus, sendo então essa posição adequada por facilitar o acesso do canal vaginal, além de diminuir a ação da força da gravidade que atua no assoalho pélvico, de maneira a não interferir nas mensurações de pressões.

Após posicionar a paciente, a mesmas foi submetida a um treinamento prévio, realizando duas contrações da musculatura do assoalho pélvico (MAP), para conscientização das manobras a serem solicitadas, sob supervisão e voz de 
comando da pesquisadora. Visando identificação da pressão perineal de forma isolada, a qual se faz necessária devido o recrutamento automático das musculaturas adjacentes, principalmente a abdominal e adutora, quando solicitado o MAP.

Com o treinamento pélvico feito, iniciou-se o AFA, no qual a terapeuta orienta que a paciente realize uma contração máxima como se fosse prender a urina, com isso é possível verificar o grau de contração dos músculos pélvicos, de acordo com a escala de avaliação funcional do assoalho pélvico, a partir das seguintes classificações, grau 0 quando há função perineal objetiva; grau 1 reconhecível somente à palpação; grau 2 contração fraca à palpação; grau 3 resistência opositora à palpação, não mantida; e grau 4 resistência opositora > 5 segundos (ORTIZ et al, 1996).

Realizado o treinamento de conscientização, a sonda de látex previamente higienizada álcool 70\%, revestida com um preservativo não lubrificado Olla (Micritex), recoberto por lubrificante vaginal $\mathrm{KY}$, a mesma foi introduzida lentamente pela vagina.

Após esta etapa, insuflou-se o aparelho até a percepção da presença da sonda insuflada no canal vaginal, logo após solicitou-se uma contração voluntária máxima realizada na expiração, de forma a registrar este valor (pressão máxima) para assim obter o valor da pressão perineal. Esta mensuração feita duas vezes, respeitando-se um período de repouso de aproximadamente 15 segundos entre uma e outra, para assim registrar o valor a ser considerado neste estudo que será o maior resultado obtido. Após as mensurações foi desinflado, retirado do canal vaginal e descartada.

$\mathrm{Na}$ pesquisa utilizou-se no modo não informatizado, em uma torre de Bargraph (barra de luzes coloridas) acessório do equipamento. Deste modo, foram visualizadas as luzes de acordo com as forças das contrações perineais, através da torre de Bargraph, mensuradas em $\mathrm{mmHg}$, por um manômetro de pressão acoplado ao aparelho.

Os dados foram analisados $\mathrm{e}$ resultaram em quadros que, posteriormente, foram sistematizados em gráficos, com suporte do programa Microsoft Office Excel 2007 e do software BioEstat na versão 5.0. Foi estabelecido o nível de significância alfa $=0.05$ para rejeição da hipótese de nulidade. A análise comparativa entre o grupo de Mulheres Sedentárias e o grupo de Mulheres Praticante de Atividade Física foi realizada 
pelo Teste U de Mann-Whitney para as variáveis ordinais e quantitativas.

\section{RESULTADOS E DISCUSSÃO}

O assoalho pélvico feminino está dividido em três porções sendo elas estruturas de sustentação. Quanto à composição das fibras, $70 \%$ são do tipo I (lenta) e $30 \%$ do tipo II (rápida). Sendo essas estruturas primordiais no suporte e manutenção dos órgãos pélvicos nas condições fisiológicas, e por serem interligadas há uma maior probabilidade de dificuldade na identificação de fraqueza do assoalho pélvico (GLISOI, GIRELLI, 2011).

Um assoalho pélvico com diminuição de força pode ser um fator para a incontinência urinária e outras patologias relacionadas à musculatura perineal. Desta forma, para ter sucesso no ganho de força da musculatura do assoalho pélvico, a conscientização da região perineal e o fortalecimento do mesmo tornam-se importante (DINIZ et al, 2014).

A pesquisa teve como amostra inicial de 12 mulheres, sendo duas exclusas devido ao período menstrual. A casuística final de dez mulheres, sendo a média de idade de 22 anos, e divididas em dois grupos, quatro sedentárias e seis praticantes de atividade física, há pelo menos seis meses. Dentre essas seis apresentaram como problema ginecológico a infecção urinária, e relataram não ter realizado nenhuma cirurgia pélvica.

Foram excluídas mulheres que tiveram alguma gravidez, com diagnóstico ou sequela de doenças ginecológicas, usuárias de medicamentos (exceto anticoncepcional) e as que utilizavam marca passo, ou seja, cardíacas.

Quando questionadas sobre a perda de urina ao realizarem grandes esforços, como tossir, espirrar, rir, ou durante a atividade física, apenas uma voluntária do grupo de praticantes de atividade física constatou positivo para perda em grandes esforços.

Ao perguntar para o grupo de praticantes de atividade física, sobre a frequência de treinos semanais, quatro responderam que praticam três vezes por semana e apenas duas praticam mais de três vezes por semana, assim como o tempo de treino por dia, apenas uma pratica no tempo de duas horas, enquanto as outras praticam 1 hora por dia.

A partir da avaliação da força muscular do assoalho pélvico, foi possível identificar a média dos valores nos dois grupos de mulheres avaliadas, apresentando durante o AFA grau de força 4 para as praticantes e 3 para as 
sedentárias, no Biofeedback força máxima $2,9 \mathrm{mmHg}$ para as praticantes, enquanto as sedentárias apresentaram 2,3 $\mathrm{mmHg}$, e as resistências foram de 3,5 segundos e 2 segundos de contração, respectivamente.

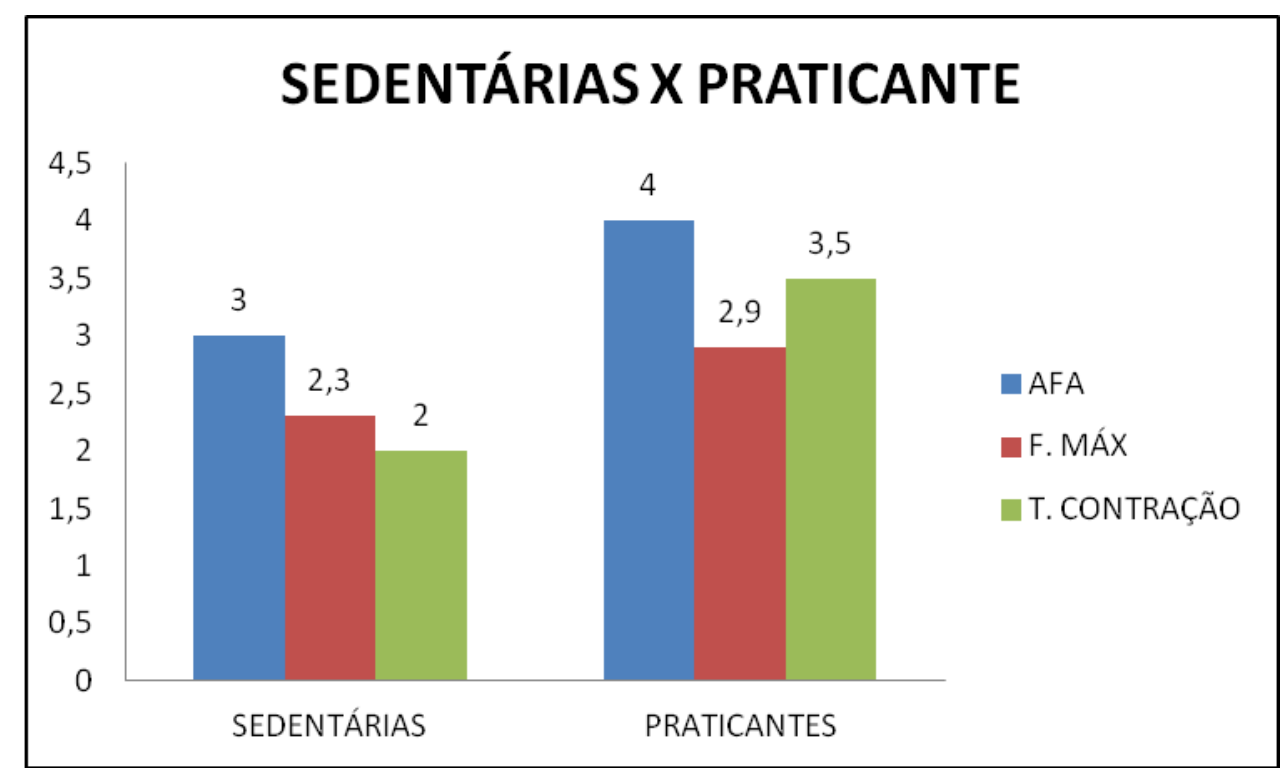

Gráfico 1. A comparação de força do assoalho pélvico em mulheres sedentárias e praticantes de atividade física.

Fonte: Pesquisa de campo, 2015.

p-valor $=0.0275$

Por meio das amostras, obtiveramse os resultados do teste de Mann- Whitney com o p-valor $=0.0275$. Portanto é possível afirmar que a força muscular do assoalho pélvico das mulheres sedentárias é inferior às praticantes com diferença estatisticamente significante.

O estudo de Silva e Moraes (2006) onde foi utilizado o AFA como método de avaliação da força, mostrou que as mulheres sedentárias possuem grau de força de contração muscular do assoalho pélvico maior que as mulheres praticantes de atividade física, o que vai de encontro então com o atual resultado.

Brinck e Nery (2006) afirma que quando a musculatura do assoalho pélvico é submetida a sobrecarga, forçada a trabalho extra para superar uma resistência ou carga, este trabalho conduz a um aumento de força, pois o músculo se contrai mais vigorosamente e a síntese de proteínas musculares é estimulada. Após um período de descanso e recuperação novas proteínas são construídas tornando as fibras musculares maiores em diâmetro e força. 
Porém na pesquisa de Alves et al (2011) demonstrou que na contração voluntária (a partir da função eletromiográfica) as sedentárias avaliadas tinha uma média de contração de 19,2 $\mathrm{CmH} 2 \mathrm{O}$ contra 22,6 cmH2O das praticantes de atividade física.

Thakar (2000) afirma que praticantes de atividade podem gerar uma dissinergia abdômino-pélvica o que seria um fator predisponente à fraqueza muscular de assoalho pélvico, em contra partida outros autores afirmam que essa sobrecarga geraria aquisição de força.

Rothstein (1982), explica que o sedentarismo, a obesidade, o confinamento ao leito, e a obstipação também leva à atrofia de fibras de contração rápida.

Sendo assim, para verificar se houveram interferências entre as variáveis, como peso e idade, no resultado, foi feito uma comparação entre os mesmos com três tipos de forças avaliadas no estudo.

$\mathrm{O}$ fator idade, principalmente na mulher tende ser a causa da diminuição de força pélvica devido a alterações como diminuição de fibras colágenas e diminuição de capacidade de armazenamento da bexiga (VIRTUOSO et al, 2011).

A partir da análise do gráfico abaixo, da idade em relação à força muscular, verificou-se que a média da força muscular do grupo de sedentárias por meio do AFA, foi de grau 4 para a média de idade de 22 anos, e grau 3 para a média de idade de 34 anos, assim como, tiveram força máxima de 2,9 e 2,3 mmHg e contração de $\quad 1,5$ e 2 segundos respectivamente.

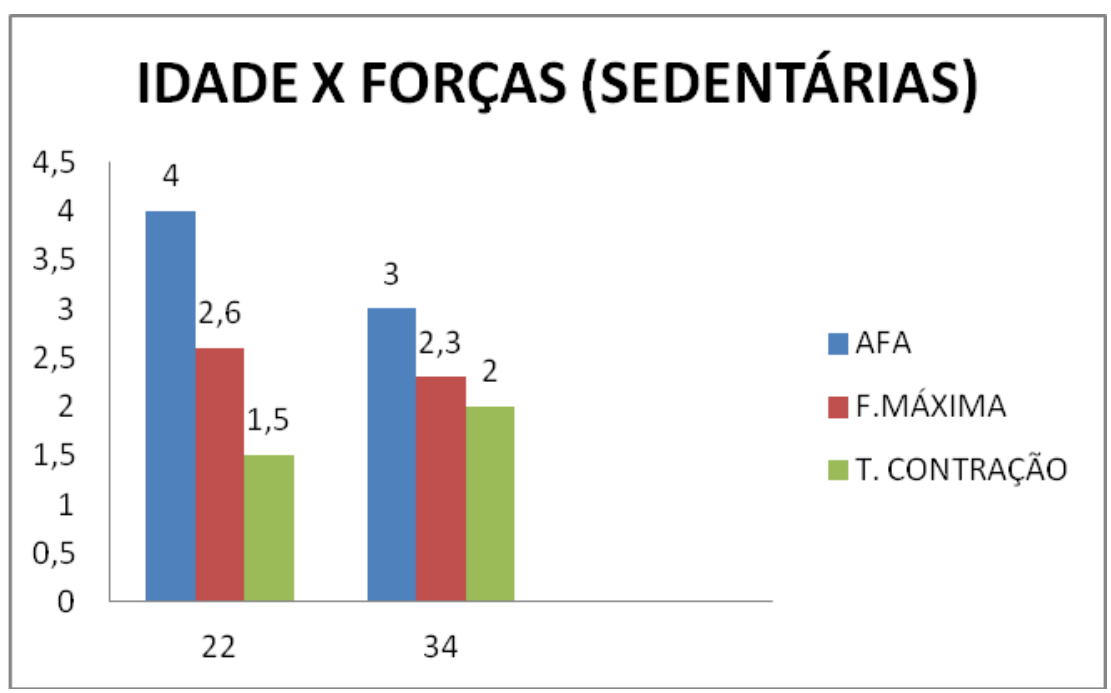

Gráfico 2. Avaliação da idade e força do assoalho pélvico em mulheres sedentárias. Fonte: Pesquisa de campo, 2015. p-valor $=0.0105$ (Mann- Whitney) 
A partir do resultado do $\mathrm{p}$-valor $=$ 0.0105 é possível identificar que a relação entre a idade e a força foi estatisticamente significante, mostrando então que conforme a idade for aumentando ocorre uma alteração da força muscular do assoalho pélvico.

No gráfico da idade em relação à força muscular, observou-se que a média do grupo de praticantes através do AFA, foi de grau 4, assim como, tiveram força máxima de 2,9 em todas as idades e contração de 4 segundos nas mulheres com 22 anos, 2 segundos nas de 26 anos, 5 segundos nas de 33 anos e 3 segundos nas de 20 anos.

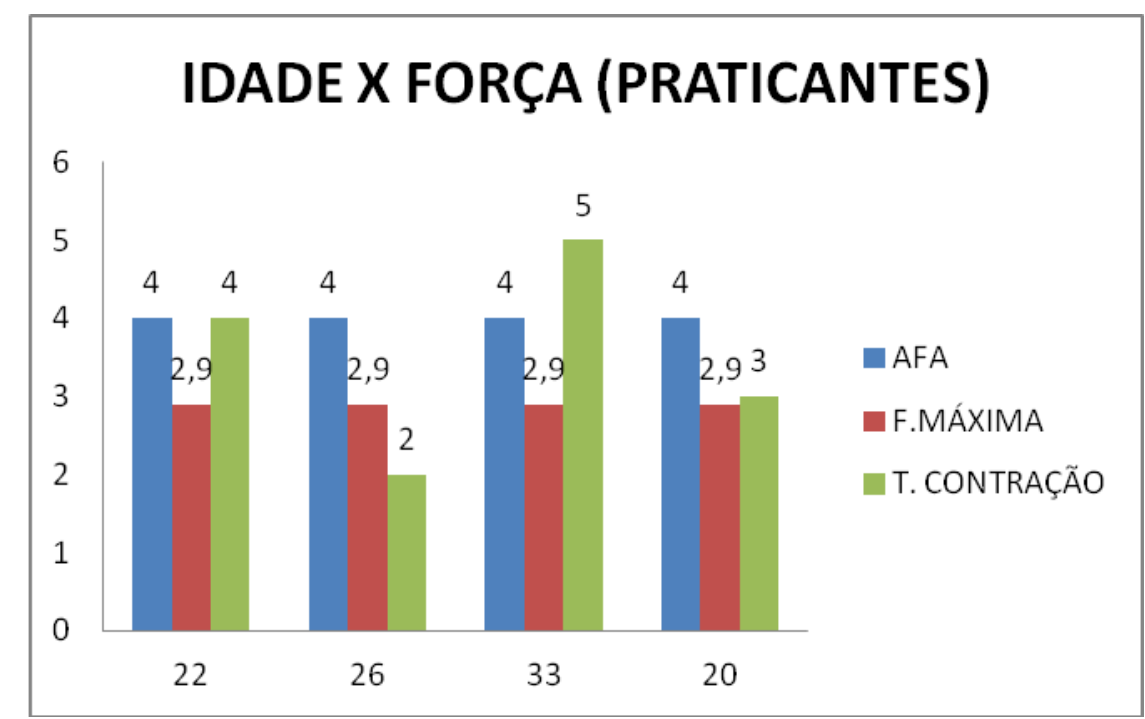

Gráfico 3. Avaliação da idade e força do assoalho pélvico em mulheres praticantes de atividade física. Fonte: Pesquisa de campo, 2015. p-valor $=0.0105$ (Mann- Whitney)

Sendo assim, em mulheres observado no gráfico em tempo de sedentárias, a idade interfere diretamente com a força muscular do assoalho pélvico, contração.

Segundo Virtuoso (2011) com o diminuindo o número de fibras de processo de envelhecimento, o trato contração rápida. Porém, nas mulheres urinário inferior feminino apresenta praticantes avaliadas com maior idade, houve um aumento do tempo de contração da musculatura pélvica, podendo ser algumas alterações, tais como atrofia muscular devido à deficiência de estrógenos, substituição de tecido muscular 
por tecido adiposo e consequente diminuição da força de contração dos músculos do assoalho pélvico.

Moreira e Arruda (2010) afirmam que em mulheres mais velhas ocorrem uma redução de colágeno na fáscia pélvica, associado com o aumento de pontes cruzadas de tecido fibroso, o que diminui a elasticidade e, portanto, a força muscular, fator que não está diretamente relacionado ao tempo de contração, como mostra a pesquisa.

Outra variável que interfere com a análise de força da musculatura do assoalho pélvico, é o peso, onde, quando há um acumulo excessivo de gordura corporal, ocorre um aumento da pressão intra-abdominal, gerando uma sobrecarga à musculatura, comprometendo a sustentação pélvica, contribuindo então para que haja um suporte anatômico incorreto (SILVA et al, 2011).

Com a sobrecarga constante enfraquecendo e causando fadiga aos músculos pélvicos, o assoalho perde então o seu papel de atribuir ao suporte das vísceras, da continência urinária e fecal, gerando mudanças na fisiologia de estruturas musculares e fáscias da região, e as conduzindo a defeitos pélvicos de sustentação e deficiência da musculatura do assoalho pélvico (SARTORI et al, 2011).

Segundo Fitz et al (2012) o sobrepeso e a obesidade são relatados como importantes fatores de risco para o desenvolvimento de disfunções do assoalho pélvico (AP) feminino. A base fisiopatológica da relação entre obesidade e disfunções do AP é a correlação entre o índice de massa corporal (IMC) e a pressão intra-abdominal. Sugere-se que o sobrepeso e a obesidade podem estressar o AP pelo aumento de pressão intraabdominal cronicamente.

Ambas as condições, obesidade e disfunções do assoalho pélvico, impactam negativamente na qualidade de vida das mulheres que as apresentam, surgindo então a necessidade da avaliação da correlação entre o peso das avaliadas com a força da musculatura pélvica, tanto do grupo de sedentárias, quanto de praticantes. 


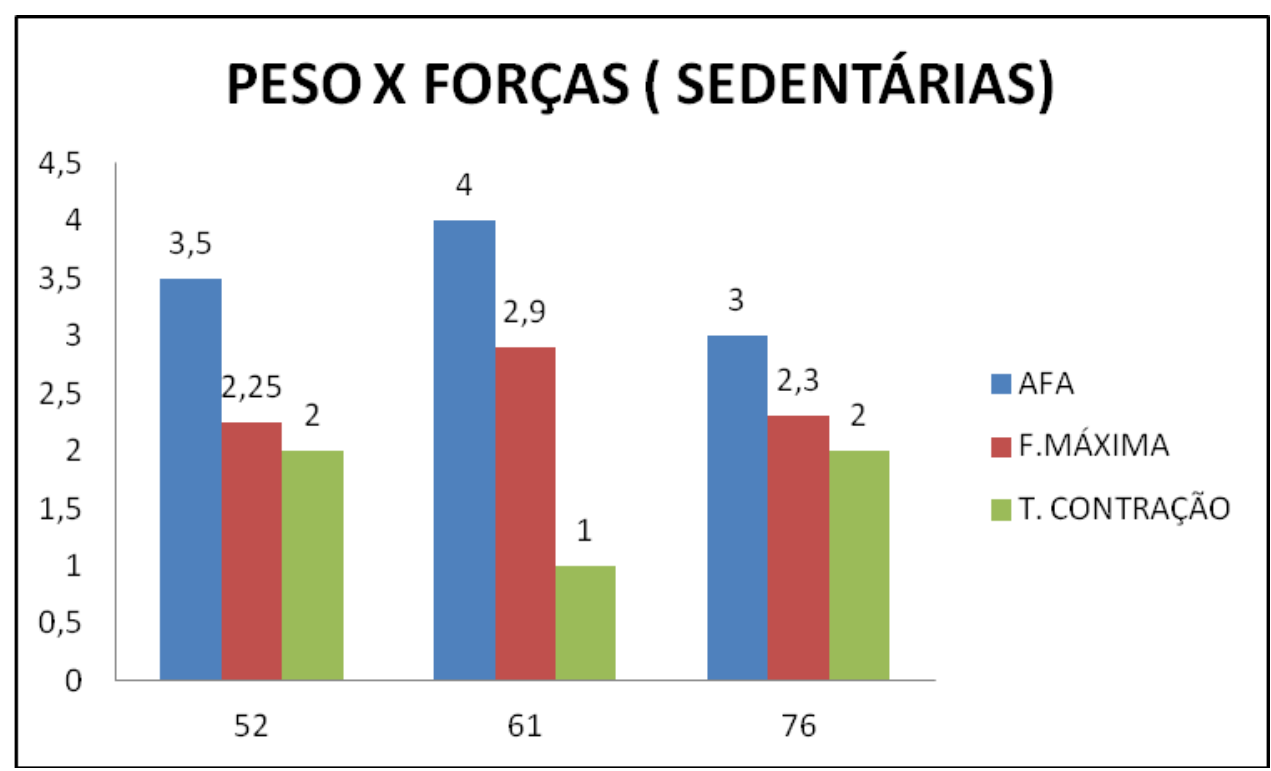

Gráfico 4. Avaliação do peso e força do assoalho pélvico em mulheres sedentárias.

Fonte: Pesquisa de campo, 2015.

p-valor $=0.0105$ (Mann- Whitney)

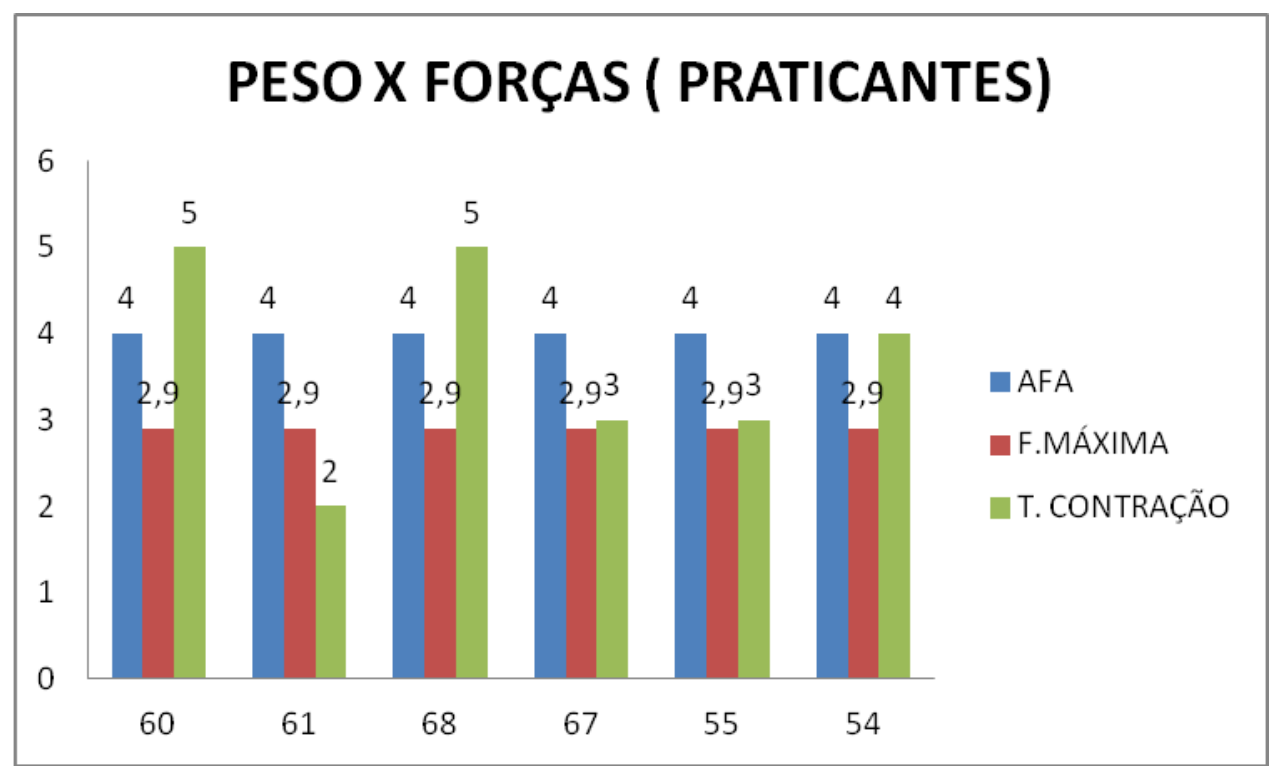

Gráfico 5. Avaliação do peso e força do assoalho pélvico em mulheres praticantes de atividade física. Fonte: Pesquisa de campo, 2015.

p-valor $<0.005$ (Mann- Whitney)

De acordo com p-valor $=0.0105$ e p-valor $<0.005$, pode-se observar que o peso também é uma variável que pode interferir diretamente na força do assoalho pélvico, havendo uma significância estatística. Porém, ao analisar os gráficos 4 e 5, notou-se, que não houve interferência em relação do peso com a força muscular, devido todas as participantes estarem do 
padrão normal do IMC (Índice de Massa Corpórea).

Nos gráficos acima, apenas uma das amostras estava no grau de sobrepeso, mesmo assim podemos verificar que não houve significância nos resultados obtidos.

Segundo Silva et al (2011), o sobrepeso é um importante fator predisponente para diminuição de força do assoalho pélvico, visto que trás modificações no posicionamento da bexiga, fazendo com que ocorra alteração da posição uretal, e diminuindo a resistência do mesmo e ocasionando não só o enfraquecimento do assoalho pélvico, como também gerando perda de urina.

A atividade física age positivamente no mecanismo de fortalecimento muscular, pois exerce forte influência na manutenção do peso corporal além de prevenir a obesidade, uma vez que a gordura adiposa pode causar elevação crônica da pressão intra-abdominal, enfraquecendo as estruturas de suporte dos músculos pélvicos (DANFORTH 2007; TOWNSEND 2008).

\section{CONCLUSÃO}

Verificou-se, que há diferença de força dos músculos do assoalho pélvico, entre mulheres sedentárias e praticantes de atividade física, pois no presente estudo, as mulheres do grupo de praticantes, obtiveram resultados superiores de grau de força durante a avaliação das mesmas, sendo então observado diferentes tipos de força, como AFA, força máxima e resistência.

As variáveis sugerem existir distinção entre as variáveis observadas, quando relacionadas ao peso e idade das avaliadas, onde mulheres mais jovens e dentro do seu peso ideal, conquistaram melhor desempenho de força da musculatura pélvica.

Este estudo obteve resultados que permitem caracterizar que mulheres praticantes de atividades físicas regulares, e de pouco impacto, como musculação, tendem a uma maior resistência e força da musculatura pélvica, desde que estejam sem grau de sobrepeso, e sejam jovens.

As mulheres atualmente estão à procura de mais conhecimento científico e de saúde, objetivando uma melhor qualidade de vida, logo, tendem a ter maior conhecimento do seu próprio corpo, e do que podem realizar para prevenção de patologias

Porém, acredita-se ainda em uma grande deficiência no que diz respeito a informações e orientações para mulheres saudáveis, no qual optam ao sedentarismo, negligenciando sua saúde e bem estar. 
Visto que, os exercícios físicos são comprovadamente benéficos à saúde, e que reduzem o risco de diversas doenças. Sendo que novos estudos com outras populações e/ou um número maior poderão ratificar os resultados encontrados e desta forma novos estudos na área poderão favorecer as mulheres quando a prevenção de alterações do assoalho pélvico.

\section{REFERENCIAS}

ALVES et al. Estudo comparativo da atividade eletromiográfica dos músculos do assoalho pélvico entre mulheres irregularmente ativas e praticantes de musculação. EFDeportes.com, Revista Digital. Buenos Aires, ano 15, n. 152, janeiro de 2011.

ALMEIDA, et al. Disfunções de assoalho pélvico em atletas. Revista feminina, v.11, n.8, agosto 2011.

BERQUO, et al. FISIOTERAPIA NO TRATAMENTO DA INCONTINENCIA URINARIA FEMININA. Revista Feminina, julho 2009. V. 37, n.7.

BRINCK, N. B; NERY, T. P. O treinamento de potência muscular de membros inferiores e a possibilidade de saltos em bailarinos contemporâneos. Revista Digital Vida e Saúde, Juiz de Fora, v1, n2, out/Nov. 2006.

\section{DANFORTH K.N, SHAH A.D, TOWNSEND MK, LIFFORD KL,} CURHAN GC, RESNICK NM, et al. Physical activity and urinary incontinence among healthy, older women. Obstet Gynecol. 2007;109(3):721-7.

DINIZ, et al. Avaliaçao da força muscular do assoalho pelvico em mulheres praticantes de mat pilates. Manual Therapy, posturology e rehabilitation jornal, 2014. 12:106-420.

FITZ, et al. Efeito da adição do biofeedback ao treinamento dos músculos do assoalho pélvico para tratamento da incontinência urinária de esforço. Rev. Bras. Ginecol. Obstet. vol.34 no.11 Rio de Janeiro Nov. 2012.

FITZ, et al. Qual o índice de massa corporal de mulheres com disfunções dos músculos do assoalho pélvico que procuram tratamento fisioterapêutico? Fisioterapia Pesquisa, 2012;19(4):309-313.

GLISOI, S. GIRELLI, P. Importancia da fisioterapia na conscientizacão e aprendizagem da contracão da musculatura do assoalho pélvico em mulheres com incontinencia urinária. Revista Brasileira de Clinica Medica, Santo Andre, SP. ovdez;9(6):408-13 .

MOREIRA, E. ARRUDA,P. Força muscular do assoalho pelvico entre mulheres continentes jovens e climatericas. Semina: Ciencias Biologicas e da saude, Londrina. V.31, n.1, p.53-61 jan/jun 2010.

MORENO A.L. Fisioterapia em uroginecologia. São Paulo: Manole, 2004.

PINHEIRO, SOUZA. Efeito da conscientizaçãoo corporal, eletroestimulação e da associação de ambas as técnicas para capacitação funcional do assoalho pélvico. 2011. $24 \mathrm{f}$. Faculdade de Ciências da Educação e da Saúde curso de fisioterapia . Centro Universitário de Brasília - UNICEUB faces -. Brasília, 2011.

REIS et al. Estudo comparativo da capacidade de contração do assoalho pélvico em atletas de voleibol e basquetebol. Rev Bras Med 
Esporte vol.17 no.2 São

Paulo Mar./Apr. 2011.

SARTORI, D. A influência da obesidade na musculatura do assoalho pélvico em mulheres continentes. Ensaios e Ciência Ciências Biológicas, Agrárias e da Saúde Vol. 15, N. 3, Ano 2011.

SILVA, A.M; LEME, A. Fortalecimento do assoalho pélvico através da dança do ventre. Nova Fisio, Revista digital. Rio de janeiro, ano 2015, n.86

SILVA, D. MORAES, N. Estudo comparativo da força muscular do assoalho pélvico em mulheres sedentárias e mulheres que praticam atividade física . Universidade da Amazônia, Para, 2006.

SILVA, et al. Grau de força muscular do assoalho pelvico em mulheres incontinentes obesas e nao obesas. Revista ciência e saúde, Porto Alegre, v.4, n.2, p. 37-44, jul-dez. 2011.
SOUZA et al. Avaliação da força muscular do assoalho pélvico em idosas com incontinência urinária. Fisioter. Mov., Curitiba, v. 24, n. 1, p. 39-46, jan./mar. 2011

TOWNSEND MK, DANFORTH KN, ROSNER B, CURHAN GC, RESNICK NM, GRODSTEIN F. Physical activity and incident urinary incontinence in middleaged women. J Urol. 2008;179(3):1012-7.

THAKAR, R. STANTON, S.L. Weakness of the pelvic floor: urological consequences. HOSPITAL MEDICINE 2000; 61(4):259-66.

VIRTUOSO, J. MAZO, G. MENEZES, E. Incontinência urinaria e força muscular perineal em idosas praticantes e não praticantes de atividade física regular. Revista Brasileira de Fisioterapia, Sao Carlos. v.14, n.4, p. 310-7, jul-agos. 2011.

ZUCCHI, E.V.M. et al. Impacto da atividade esportiva no assoalho pélvico Femina; 31, 4: 333-335, Maio 2003. 